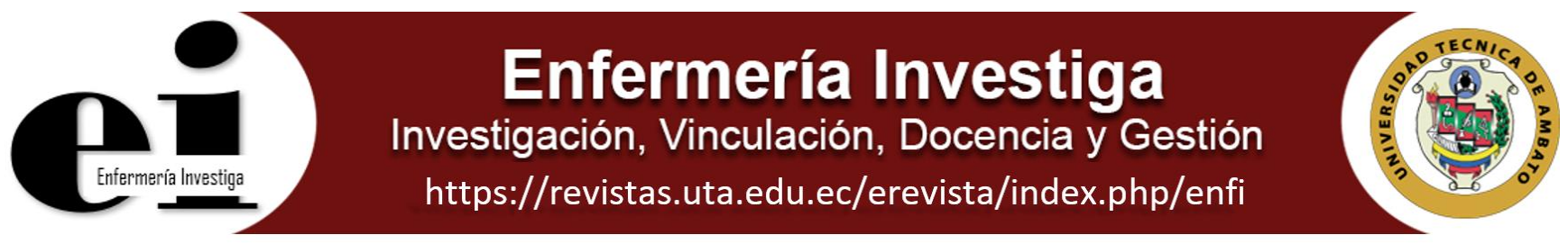

\title{
TRAQUEOSTOMÍA TEMPRANA VS TARDÍA EN PACIENTES NEUROQUIRÚRGICOS INTERNADOS EN UNA UNIDAD DE CUIDADOS INTENSIVOS.
}

\section{EARLY VERSUS LATE TRACHEOSTOMY IN NEUROSURGICAL PATIENTS RECEIVED IN AN INTENSIVE CARE UNIT}

Freiser Eceomo Cruz Mosquera1, https://orcid.org/0000-0001-7584-4636, Nathaly Erazo Builes ${ }^{2}$ https://orcid.org/0000-0003-23302127, Juan Camilo Angulo Cano ${ }^{3}$ https://orcid.org/0000-0002-0806-9227, María Paula Solarte-Roa ${ }^{4}$ https://orcid.org/0000-0003-22584599, Daniel Mauricio Muñoz Piamba ${ }^{4}$ https://orcid.org/0000-0003-1744-8322, Laura Rojas Anacona ${ }^{4}$ https://orcid.org/0000-00032097-4737.

\footnotetext{
${ }^{1}$ Investigador asociado Minciencias, Director Grupo de Investigación en Salud Integral. Universidad Santiago de Cali, Colombia.

${ }^{2}$ Terapeuta Respiratorio. Semillero de investigación SISARE. Centro de Salud Luis H. Garcés ESE Centro, Colombia.

${ }^{3}$ Terapeuta Respiratorio. Semillero de investigación SISARE. Unidad de cuidados intensivos Clínica Versalles, Colombia.

${ }^{4}$ Terapia Respiratoria. Semillero de investigación SISARE. Universidad Santiago de Cali, Colombia.
}

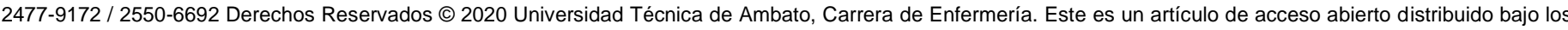

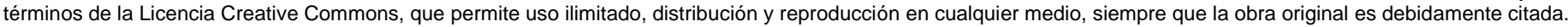

Recibido: 15 de octubre 2020

Aceptado: 27 de noviembre 2020

\section{RESUMEN}

Introducción: Los pacientes neurocríticos por lo general requieren periodos largos de ventilación mecánica, en ese contexto la traqueostomía es un procedimiento frecuente que se realiza para facilitar el destete de la ventilación y se asocia a múltiples beneficios; sin embargo, el momento de su realización sigue siendo objeto de debate. Objetivo: determinar los beneficios clínicos de la traqueostomía temprana vs la tardía en los pacientes neurocríticos que ingresan a una unidad de cuidados intensivos (UCI) polivalente de una institución de salud de la ciudad de Cali. Metodología: investigación observacional, descriptiva, de serie de casos que incluyó pacientes neuroquirúrgicos, mayores de edad que ingresaron a una $\mathrm{UCI}$ durante el periodo 2016 -2018; a partir de la muestra total se estipularon dos grupos: traqueostomía temprana ( $\leq 9$ días) y traqueostomía tardía ( $\geq 10$ días). El análisis estadístico se realizó en el programa SPSS versión 24. Resultados: Se incluyeron 20 sujetos con edad de $51.9 \pm 17$ años, 10 fueron asignados al grupo de traqueostomía temprana y 10 al grupo den traqueostomía tardía. Se evidenció que los pacientes con traqueostomía temprana tienen menos días de sedación (10 \pm 2.1 vs $16 \pm 9 ; p=0.02$ ) y los 3 casos que fallecieron habían sido traqueostomizados tardíamente. Conclusiones: La traqueostomía temprana puede traer beneficios clínicos a los pacientes neuroquirúrgicos que ingresan a unidades de cuidados intensivos.

Palabras claves: Traqueostomía, unidades de cuidados intensivos, paciente, ventilación mecánica.

\begin{abstract}
Introduction: Neurocritical patients generally require long periods of mechanical ventilation. In this context, tracheostomy is a frequent procedure performed to facilitate weaning from ventilation and is associated with multiple benefits; however, the timing of its implementation remains under debate. Objective: to determine the clinical benefits of early vs late tracheostomy in neurocritical patients admitted to a polyvalent intensive care unit (ICU) of a health institution in the city of Cali. Methodology: observational, descriptive investigation of a series of cases that included neurosurgical patients, of legal age who were admitted to an ICU during the period 2016 -2018; From the total sample, two groups were stipulated: early tracheostomy ( $\leq 9$ days) and late tracheostomy ( $\geq 10$ days). Statistical analysis was performed using SPSS version 24. Results: 20 subjects with an age of $51.9 \pm 17$ years were included, 10 were assigned to the early tracheostomy group and 10 to the late tracheostomy group. It was evidenced that patients with early tracheostomy had fewer days of sedation $(10 \pm 2.1$ vs $16 \pm 9 ; p=0.02)$ and the 3 cases that died had been tracheostomized late. Conclusions: Early tracheostomy can bring clinical benefits to neurosurgical patients admitted to intensive care units.
\end{abstract}

Keywords: Tracheostomy, intensive care units, patient, mechanic ventilation. 


\section{INTRODUCCIÓN}

Los pacientes en estado crítico son aquellos que presentan alteraciones de uno o más sistemas que comprometen su vida y que hace necesaria la ejecución de intervenciones inmediatas para corregir su condición, intervenciones entre las que se encuentra la ventilación mecánica invasiva (1-3). Por las características propias de su patología, los pacientes neurocríticos son más propensos a requerir periodos largos de soporte ventilatorio, en ese contexto cobra importancia la traqueostomía (TQT), procedimiento quirúrgico que con frecuencia se realiza para facilitar el destete de la ventilación (4$6)$.

La epidemiologia de la traqueostomía en los pacientes críticos ha sido descrita por varios autores, según Morales et al. (7) 1 de cada 10 pacientes con soporte ventilatorio por más de tres días en la $\mathrm{UCl}$ requieren de una traqueostomía. Por otro lado, Raimondi et al en consonancia con otras investigaciones reportan que la prevalencia para esta intervención es alrededor del $24 \%(8,9)$.

Desde el momento de su aparición, son muchos los beneficios que se han relacionado con la realización de la traqueostomía en pacientes neurocríticos, entre ellos disminución significativa de los días de ventilación, reducción de la estancia en cuidados intensivos, disminución de los días de hospitalización y la tasa de infecciones pulmonares (10-12). A nivel mundial diversos investigadores han desarrollado y publicado investigaciones con el propósito de determinar los beneficios y complicaciones relacionadas con la traqueostomía temprana vs tardía, sin embargo, los hallazgos siguen siendo controversiales.

Arrona et al. evaluaron una cohorte de 42 pacientes que requirieron traqueostomía como alternativa, para una posible ventilación mecánica prolongada. Se consideraron TQT tempranas aquellas que se realizaron en menos de 14 días de intubación orotraqueal y tardías de 14 o más días. La realización de traqueostomía en ambos grupos fue realizada en el quirófano, lo que entre otras cosas incrementó el riesgo de infección por los traslados, tamaño de la herida y el uso de puntos de fijación en aquellas incisiones que fugaron (13).

Por otro lado, Neupane et al. desarrollaron un estudio para evaluar la efectividad de la traqueostomía temprana y de la traqueostomía tardía en pacientes críticos de neurocirugía. Se incluyeron 30 pacientes neurocriticos y se dividieron en 2 grupos (traqueostomía temprana $<7$ días, traqueostomía tardía $>7$ días) posterior a un riguroso análisis encontraron que los pacientes a quienes la intervención se les realizaba antes de los 7 días tenían una estadía más corta en UCl y los días de ventilación mecánica, pero no afecta la tasa de mortalidad y complicaciones relacionadas con el procedimiento (14). Zirpe et al. describen los beneficios de la traqueostomía temprana en pacientes con traumatismo craneoencefálico severo, que requieren de apoyo mecánico ventilatorio prolongado, resultando la traqueostomía un procedimiento de elección favorable para disminuir la intubación prolongada minimizando significativamente las secuelas de la misma (15).

A pesar de los beneficios descritos, el momento de realización de la traqueostomía para garantizarlos sigue siendo objeto de debate, por esta razón se plantea la presente investigación con el objetivo de determinar los beneficios clínicos de la traqueostomía temprana ( $\leq 9$ días) vs la traqueostomía tardía ( $\geq 10$ días) en los pacientes neuroquirúrgicos que ingresaron a una UCI de una institución de salud de la ciudad de Cali.

\section{MÉTODOS}

Estudio observacional descriptivo de serie de casos en el que se incluyeron un total de 20 pacientes neurocríticos traqueostomizados, que fueron internados en una $\mathrm{UCI}$ polivalentes de una Clínica de alta complejidad, en el periodo comprendido entre enero de 2016 y diciembre 2018. Para efectos de comparación los pacientes se distribuyeron en dos grupos: aquellos traqueostomizados en los primeros 9 días de intubación (traqueostomía temprana) y los traqueostomizados en un tiempo $\geq 10$ días (traqueostomía tardía). Se incluyeron los pacientes mayores de 18 años a quienes la traqueostomía se les realizó durante su estancia en $\mathrm{UCl}$ en el periodo de estudio y se excluyeron los sujetos con información insuficiente en las historias clínicas.

De cada participante se registraron variables sociodemográficas y clínicas como edad, sexo, servicio de ingreso al centro de atención, diagnóstico principal, lugar en el que se realizó la intubación orotraqueal, medición de la gravedad y probabilidad de mortalidad por medio de la escala de Acute Physiology And Chronic Health Evaluation II (APACHE), aplicación de la escala coma de Glasgow en la valoración de ingreso a $\mathrm{UCl}$, días de sedación y manejo antibiótico relacionado estrictamente con el tratamiento de una infección pulmonar. Se contemplaron variables relacionadas con el procedimiento de estudio, como tipo de traqueostomía (quirúrgica y percutánea) causa del procedimiento y momento de realización (en temprano y tardío). Además, días de ventilación mecánica posterior a la traqueostomía, desarrollo de neumonía asociada a la ventilación mecánica, estancia en UCl y hospitalaria, y estado al egreso del hospital.

\section{Análisis estadístico.}

A partir de la información registrada en la base de datos de Excel 2007 se realizó el análisis estadístico en el programa IBM SPSS 24. Inicialmente se estableció la normalidad de la distribución de las variables cuantitativas usando la prueba de Shapiro Wilk. Las variables continuas se expresaron como promedio o mediana, con sus respectivas medidas de dispersión para determinar la variabilidad de la distribución (desviación estándar). Las variables cualitativas se expresaron como valor absoluto y porcentaje.

Para realizar comparación de medias entre grupos se utilizó la prueba t de Student para muestras independientes y U de Mann Whitney de acuerdo a la normalidad de la distribución. A fin de establecer la diferencia de proporciones se usó el test de Chi cuadrado de Pearson considerando en ambos escenarios una $\mathrm{p}<0.05$ como estadísticamente significativa.

\section{Aspectos éticos.}

La investigación siguió los lineamientos éticos de la resolución 8430 del Ministerio de salud de Colombia y de la declaración del Helsinki; el protocolo contó con el aval ético de la Universidad Santiago de Cali y la Clínica en la que se ejecutó la investigación. 


\section{RESULTADOS.}

Un total de 20 pacientes fueron identificados (traqueostomía tardía: 11 y traqueostomía temprana: 9) con una edad promedio de $51,9 \pm 17$ años y en su mayoría de género masculino $(78 \%$ y $45 \%$ respectivamente). No se identificaron diferencias significativas entre grupos en gran parte de las variables clínicas salvo en los días de intubación hasta la traqueostomía $(7,1 \pm 1,3$ vs $11,5 \pm 1,7 ; p=0,001)$; respecto al puntaje de la escala coma de Glasgow medida al ingreso a la $\mathrm{UCl}$, si bien fue similar (8 vs 9 $p=0,4)$ se evidenció que la frecuencia de presentación de un puntaje $<8$ fue superior en el grupo de traqueostomía temprana $(78 \%)$ que en el de traqueostomía tardía (54\%). (Tabla 1).

Tabla 1. Comparación de variables sociodemográfica y clínicas de los grupos

\begin{tabular}{|c|c|c|c|}
\hline Variable & $\begin{array}{c}\mathrm{TQT} \leq 9 \text { dias } \\
(\mathrm{n}=9)\end{array}$ & $\begin{array}{c}T Q T \geq 10 \text { dias } \\
(n=11)\end{array}$ & Valor $\mathrm{p}^{*}$ \\
\hline Edad & $55,4 \pm 15,8$ & $52,6 \pm 15,9$ & 0,7 \\
\hline \multicolumn{4}{|l|}{ Sexo } \\
\hline Femenino & $2(22 \%)$ & $6(55 \%)$ & \multirow{2}{*}{0,14} \\
\hline Masculino & $7(78 \%)$ & $5(45 \%)$ & \\
\hline Glasgow de ingreso & $8 \pm 3,1$ & $9 \pm 3,2$ & 0,4 \\
\hline APACHE de ingreso & $29 \pm 5,4$ & $30 \pm 3,2$ & 0,8 \\
\hline \multicolumn{4}{|l|}{ Servicio de ingreso } \\
\hline Urgencias & $8(89 \%)$ & $9(82 \%)$ & \multirow{2}{*}{0,6} \\
\hline Otros & $1(11 \%)$ & $2(18 \%)$ & \\
\hline \multicolumn{4}{|l|}{ Tipo de enfermedad } \\
\hline Vascular & $6(67 \%)$ & $5(46 \%)$ & \\
\hline Tumores & $2(22 \%)$ & $3(27 \%)$ & 0,57 \\
\hline Traumática & $1(11 \%)$ & $3(27 \%)$ & \\
\hline Intubación orotraqueal hasta la TQT (días) & $7,1 \pm 1,3$ & $11,5 \pm 1,7$ & 0,001 \\
\hline
\end{tabular}

*U de Mann Whitney, Chi 2 de Pearson.

El área del hospital en la que los pacientes fueron intubados con mayor frecuencia fue el servicio de urgencias (60\%) seguido de quirófano (35\%) y UCI (5\%).

Con relación a la causa de traqueostomía, en ambos grupos se evidencia que en la totalidad de los sujetos se realizó por una lenta evolución clínica, de acuerdo al tipo de traqueostomía se encuentra que en ambos grupos la TQT percutánea fue más frecuente que la quirúrgica (56\% en el grupo de TQT temprana y $64 \%$ en grupo de TQT tardía) (figura 1).

Figura 1. Tipo de traqueostomía en la población a estudio.

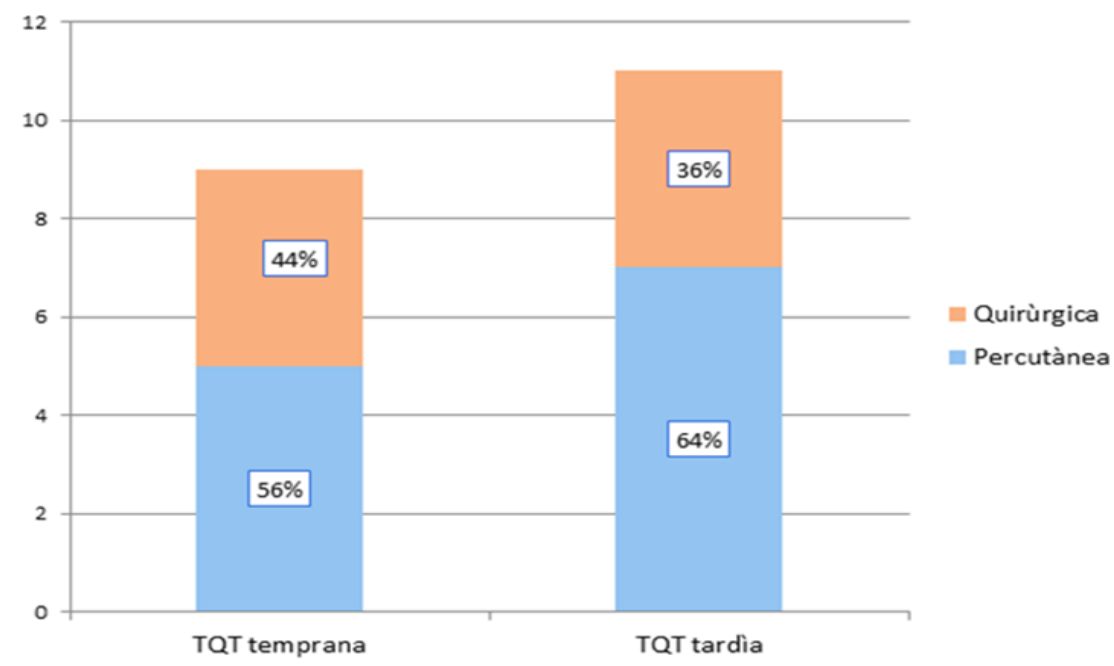


Respecto a los días de ventilación mecánica posterior a la traqueostomía, se observa que no hay diferencias significativas entre los grupos de TQT temprana vs tardía $(p=0,08)$. Con relación a los días de requerimiento de sedantes se encuentra que son menores en el grupo de traqueostomía temprana (10 vs $16 ; p=0,02)$. Al evaluar la frecuencia de neumonía asociada a la ventilación mecánica se halla que la diferencia es clínicamente relevante ( $11 \%$ vs $45 \%$ ) sin embargo, no es estadísticamente significativa. Referente a la estancia en la unidad de cuidados intensivos y el hospital, se encuentra que aquellos que son traqueostomizados de forma temprana tienen un comportamiento similar respecto a los traqueostomizados tardíamente (tabla 2).

\section{Tabla 2. Distribución de las características clínicas en los grupos traqueostomía temprana vs tardía.}

\begin{tabular}{lccc}
\hline \multicolumn{1}{c}{ Variable } & $\begin{array}{c}\text { TQT } \mathbf{~} \mathbf{9} \text { días } \\
\mathbf{n = 9}\end{array}$ & $\begin{array}{c}\text { TQT } \geq \mathbf{1 0 d i ́ a s} \\
\mathbf{n = 1 1}\end{array}$ & Valor $\mathbf{p}^{*}$ \\
\hline V.M postraqueostomìa (días) & $8,3 \pm 6$ & $11 \pm 8$ & 0,3 \\
Sedación (días) & $10 \pm 2,1$ & $16 \pm 9$ & 0,02 \\
NAVM & $1(11 \%)$ & $5(45 \%)$ & 0,09 \\
Estancia en UCI (días) & $19 \pm 8$ & $24,4 \pm 8$ & 0,09 \\
Estancia hospitalaria (días) & $29 \pm 7,3$ & $30,8 \pm 8,8$ & 0,82 \\
\hline
\end{tabular}

NAVM: neumonía asociada a la ventilación mecánica. V.M: ventilación mecánica.

*U de Mann Whitney

Para finalizar, los días de manejo antibiótico distaron de manera importante entre grupos ((TQT temprana 7 vs TQT tardía 15.6) y

\section{DISCUSIÓN}

En la actualidad la traqueostomía sigue siendo una alternativa de manejo importante en las distintas $\mathrm{UCl}$, si bien su uso no se circunscribe a cierto tipo de pacientes, es necesario mencionar que algunos grupos entre los que se encuentran los neurocríticos, por sus características clínicas son sometidos a esta intervención con mayor frecuencia. Los efectos clínicos atribuibles a la traqueostomía han sido ampliamente descritos, sin embargo, aún no se logra dilucidar o establecer un consenso sobre el momento oportuno para su realización, razón fundamental por la que se adelantó este estudio $(16,17)$.

Con relación al momento de la traqueostomía, en esta serie de casos se consideró traqueostomía temprana aquella realizada en un tiempo menor o igual a 9 días y traqueostomía tardía en un tiempo superior a ese término; es importante resaltar que la clasificación aquí realizada no obedece a una definición estandarizada, si bien algunos estudios lo proponen no existe un consenso establecido sobre el significado de estos dos términos (TQT temprana y TQT tardía), estando con frecuencia sujetos a la visión del grupo multidisciplinario que realiza el seguimiento a cada caso; lo anterior ha contribuido a que la agrupación de estudios al respecto resulte difícil y genere mucha más controversia $(18,19)$.

En la comparación de los grupos es necesario resaltar que no hubo diferencias estadísticamente significativas en el puntaje obtenido es las escalas de pronóstico y nivel de conciencia medidas al ingreso (APACHE II, Glasgow), hallazgo similar al encontrado por Milian et al. los cuales en un grupo de 63 pacientes divididos en TQT precoz ( $<12$ días) y tardía ( $>12$ días) no evidenciaron divergencias importantes en la puntuación de APACHE II (17,8 vs $16,9 \mathrm{p}=0,456)$ y Glasgow (9,3 vs 10,62 los tres casos que fallecieron en el periodo de observación recibieron la traqueostomía de manera tardía.

$p=0,06)$, es preciso mencionar que no se realizó un seguimiento en la puntuación de estas escalas posterior al ingreso (20).

En la investigación actual, con respecto a la causa de la traqueostomía la razón fundamental fue la lenta evolución clínica, similar Plummer y Gracey, así como Barrio et al., los cuales plantean como principales causas, la ventilación mecánica prolongada, dificultad para el destete ventilatorio y baja probabilidad de extubación; que son las más frecuentes de la evolución clínica lenta $(21,22)$

A propósito del tipo de traqueostomía el método más usado en ambos grupos fue el percutáneo, método que se convirtió desde mediado de la década de 1980 en la alternativa que se recomienda con mayor frecuencia hasta hoy, por ser mínimamente invasivo y no exigir el traslado del paciente a quirófano; sin embargo, los resultados obtenidos en estudios que comparan los dos métodos de traqueostomía (percutáneo y quirúrgico) son controversiales (23).

Con relación a los días de ventilación mecánica posterior a la traqueostomía no se presentaron diferencias significativas entre grupos (8.3 vs 11; $p=0,3$ ) aunque hubo una tendencia ligeramente menor en los pacientes con traqueostomía temprana, aspecto relevante si se tiene en cuenta que la cánula de traqueostomía genera un espacio muerto 3 a 4 veces menor que el tubo orotraqueal, lo que disminuye la resistencia, mejora notablemente la mecánica ventilatoria y reduce ligeramente la probabilidad de requerir soporte ventilatorio; a pesar de lo anterior, se debe tener en cuenta que la VM postraqueostomìa depende de muchos factores, entre ellos la condición clínica y el curso de la enfermedad de cada paciente (24).

Al evaluar la frecuencia de neumonía se evidencian diferencias clínicamente importantes, pero no estadísticamente significativas (11\% vs $45 \%$ ), lo que puede influir en los días de sedación y manejo antibiótico. Lo anterior tiene relación con lo 
reportado por Khammas et al. en una población de 67 pacientes ingresados a UCl divididos en dos grupos (TQT temprana 10 días y tardía >10 días) en los que encontraron que la neumonía asociada a la ventilación mecánica y los días de sedación fueron menores en el grupo TQT temprana (25).

Es importante tener en cuenta que, si bien el desarrollo de neumonía está relacionado con el tiempo de exposición al tubo orotraqueal, el lugar donde se realiza la intubación puede influir notablemente en el desarrollo de esta patología; en esta investigación la mayor parte de las intubaciones fueron realizadas en urgencias, seguido del quirófano y finalmente $\mathrm{UCl}$. Según lo observado con anterioridad, Tsakiridou et al. realizaron una investigación con el propósito de evaluar si la intubación previa al ingreso a UCI se asociaba con NAVM, tras analizar una población de 100 sujetos encontraron que incurrir en esta práctica aumenta la probabilidad de padecer la neumonía (26). Referente a los días de estancia en UCI y en el servicio de hospitalización general no se reportaron diferencias importantes. Lo anterior sugiere que tales eventos son multicausales y están relacionados estrechamente con otras variables como la enfermedad de base (27), es importante mencionar que si bien todos los casos que murieron estaban en el grupo de traqueostomía tardía esto no permite establecer una relación como bien lo han declarado varios estudios $(28,29)$.

Con relación a las limitaciones del estudio, es importante destacar que solo se registró el puntaje de ingreso del APACHE y el Glasgow, lo anterior no permitió hacer un seguimiento para establecer si los pacientes presentaron una mejoría de su condición, incluso en las primeras 48 horas, que claramente podría haber influido en su evolución en la UCl. Lo mencionado anteriormente es fundamental dado que algunos estudios han mostrado que los pacientes con trauma craneoencefálico pueden presentar una mejoría en el puntaje de Glasgow de hasta 2 puntos en las primeras 48 horas (30). Por otro lado, el tipo de diseño utilizado no permite recomendar ampliamente asociaciones entre el evento evaluado y algunos desenlaces.

\section{CONCLUSIONES:}

La traqueostomía es un procedimiento habitual en los pacientes neuroquirúrgicos que ingresan a $\mathrm{UCl}$ y requieren ventilación mecánica, los efectos clínicos atribuibles a la traqueostomía han sido ampliamente descritos, sin embargo, aún no se logra dilucidar o establecer un consenso sobre el momento oportuno para su realización.

Las principales causas, para la realización de traqueostomía son: la ventilación mecánica prolongada, dificultad para el destete ventilatorio y baja probabilidad de extubación.

Con relación a los días de ventilación mecánica posterior a la traqueostomía, es e resaltar que la cánula de traqueostomía genera un espacio muerto 3 a 4 veces menor que el tubo orotraqueal, lo que disminuye la resistencia, mejora notablemente la mecánica ventilatoria y reduce ligeramente la probabilidad de requerir soporte ventilatorio.

FINANCIAMIENTO: no hubo fuentes de financiamiento. CONFLICTOS DE INTERESES: Los autores declaran no tener conflictos de interés.

AGRADECIMIENTO: a todos los estudiantes de la Electiva de Profundización en Unidad de Cuidados Intensivos neuroquirúrgicos del Programa de Terapia Respiratoria de la Universidad Santiago de Cali, los cuales, con espíritu crítico y ánimo incansable, además de indagar sobre el manejo de este grupo de paciente, buscan con diligencia las respuestas a través de la investigación. A los directivos de la antigua Clínica Esimed por permitir la ejecución de esta investigación.

\section{REFERENCIAS BIBLIOGRÁFICAS}

1. Aguilar C, Martínez C. La realidad de la Unidad de Cuidados Intensivos. Med Crit 2017;31(3):171-173. Disponible en: http://www.scielo.org.mx/scielo.php?script=sci_arttext\&pid=S2448-89092017000300171

2. Gan L, Sierra R, Díaz E, Pouymirò R, Armas K. Ventilación mecánica en pacientes con hemorragia cerebral. Rev Inf Cient. 2015; 90(2):301-313. Disponible en: https://www.redalyc.org/pdf/5517/551757251011.pdf

3. Mechanical ventilation in patients with acute ischaemic stroke: from pathophysiology to clinical practice. Crit Care.2019; 23: 388.Disponible en: https://link.springer.com/article/10.1186/s13054-019-2662-8\#citeas

4. Bösel J. Use and Timing of Tracheostomy After Severe Stroke. Stroke. 2017;48(9):2638-2643. Disponible en: https://www.ahajournals.org/doi/full/10.1161/STROKEAHA.117.017794

5. Kumar V, Kiran N, Kumar V, Ghosh A, Pal R, Reddy V, et al. The Outcome Analysis and Complication Rates of Tracheostomy Tube Insertion in Critically III Neurosurgical Patients; A Data Mining Study. Bull Emerg Trauma. 2019;7(4):355-360. Disponible en:https://pubmed.ncbi.nlm.nih.gov/31857997/.

6. Schonenberger S, Niesen W ,Fuhrer H, Bauza C, Klose C, Kieser M, et al. Early tracheostomy in ventilated stroke patients: Study protocol of the international multicentre randomized trial SETPOINT2 (Stroke-related Early Tracheostomy vs. Prolonged Orotracheal Intubation in Neurocritical care Trial 2). International Journal of Stroke. 2016;11(3): 368-379. Disponible:https://journals.sagepub.com/doi/full/10.1177/1747493015616638?casa_token=B50Whad5GfkAAAAA\%3ApywL zibL3nz8I7n5KFgxJUjehNf6wQwJTV7mYh16ucYvMvCkdTZDx8Wo0ezdLxGaZNiHEMxfK65zEpM

7. Morales J, Díaz P, Cortés A. Manejo integral del paciente con traqueostomia. Neumol Cir Torax. 2014;73(4): 254-262. Disponible en: http://www.scielo.org.mx/pdf/nct/v73n4/v73n4a6.pdf

8. Raimondi N, Vial M, Calleja J, Quintero A, Cortes A, Celis E, et al. Guías basadas en la evidencia para el uso de traqueostomia en el paciente crítico. Med Intensiva. 2017; 41(2):94-115. Disponible en: http://www.enfermeriaaps.com/portal/wp-content/uploads/2017/07/Gu\%C3\%ADas-basadas-en-la-evidencia-para-el-usode-traqueostom\%C3\%ADa-en-el-paciente-cr\%C3\%ADtico.pdf

9. Baron D, Hochrleser H, Metnitz G, Mauritz W. Tracheostomy is associated with decreased hospital mortality after moderate or severe isolated traumatic brain injury. Wien Klin Wochenschr. 2016; 128: 397-403. Disponible en: 
https://www.ncbi.nlm.nih.gov/pmc/articles/PMC4916187/\#: :text=Despite\%20the\%20greater\%20severity\%20of,multifactori al\%20and\%20require\%20further\%20investigation.

10. Robba C, Galimberte S, Graziano F, Wiegers E, Lingsma C, Menon D, et al. Tracheostomy practice and timing in traumatic brain-injured patients: a CENTER-TBI study. Intensive Care Med. 2020; 46: 983-994. Disponible en: https://link.springer.com/content/pdf/10.1007/s00134-020-05935-5.pdf

11. López Y, Iglesias N, Tejidor D, Tejidor J, Moyano I. Influencia de la traqueostomía en el proceso de destete. MediCiego 2016;22(4).Disponible en: http://www.revmediciego.sld.cu/index.php/mediciego/article/view/538/1038

12. Meng L,Wang C, Li J , Zhang J. Early vs late tracheos-tomy in critically ill patients: a systematic review and meta-analysis. Clin Respir J.2016; 10: 684-692.Disponible en: https://onlinelibrary.wiley.com/doi/epdf/10.1111/crj.12286

13. Arrona $P$, Ocegueda $P$, Chávez $P$, Ramírez $M$, Nava V. Traqueostomía temprana versus traqueostomía tardía en la Unidad de Cuidados Intensivos. Med Crit. 2014;28(1):16-19. Disponible en: https://www.medigraphic.com/pdfs/medcri/ti2014/ti141c.pd

14. Neupane B, Lamichhane A. Outcome of Early versus Late Tracheostomy in Neurosurgical critical Patient. NJN.2019; 16(2):38-2. Disponible en: https://www.nepjol.info/index.php/NJN/article/view/25950.

15. Zirpe K, Tambe D, Deshmukh A, Gurav S. The Impact of Early Tracheostomy in Neurotrauma Patients: A Retrospective Study. Indio J Crit Care Med. 2017;21(1): 6-10. Disponible en: https://www.ncbi.nlm.nih.gov/pmc/articles/PMC5278595/

16. Huang W, Zhang B, Xu M, Chen G, Zhang X, Zhang J, et al. The impact of tracheostomy timing on clinical outcomes and adverse events in intubated patients with infratentorial lesions: early versus late tracheostomy. Neurosurg Rev. 2020; 1-10. Disponible en: https://www.ncbi.nlm.nih.gov/pmc/articles/PMC7314615/

17. Rodríguez E. Traqueostomía en el paciente crítico en la era del consentimiento libre Informado. Rev. bioét. $2017 ; 25$ (3): 502-11. Disponible en: https://www.scielo.br/scielo.php?script=sci_arttext\&pid=S1983$80422017000300502 \& \operatorname{lng}=e n \& n r m=i s o \& t \operatorname{lng}=e s$

18. Chen W, Liu F, Chen J, Ma L, Li G, You C. Timing and Outcomes of Tracheostomy in Patients with Hemorrhagic Stroke. World Neurosurg. 2019;131: e606-e613. Disponible en: https://pubmed.ncbi.nlm.nih.gov/31408751/

19. Durana M, Abdullayev R, Cömlekc M, Sürenc M, Bülbül M, Aldemir T. Comparación de traqueotomía percutánea precoz y tardía en unidad de cuidados intensivos para adultos. Rev Bras Anestesiol.2014.64(6):438-442. Disponible en: https://www.sciencedirect.com/science/article/pii/S2255496313001864?via\%3Dihub

20. Milian W,Contardo Z. La traqueostomía precoz mejora el pronóstico de los pacientes críticos. Hospital Nacional Edgardo Rebagliati Martins 2010; 1-9. Disponible en: https://www.clinicainternacional.com.pe/pdf/revistainterciencia/2/Articulo_Original.pdf

21. Plummer AL, Gracey DR. Concensus conference on artificial airways in patients receiving mechanical ventilation. Chest. 1989; 96:178---80. Disponible en: https://journal.chestnet.org/article/S0012-3692(15)45762-9/pdf

22. Barrio M, Berrazueta A, Pellejero J, Fernandez J, Ortiz M, Armesto D. Pacientes con indicación de traqueostomía en una cohorte de cuidados intensivos. España.Colombian Journal of Anesthesiology. 2016;44(4):278-281.Disponible en: https://www.sciencedirect.com/science/article/pii/S0120334716300806

23. Akıncı 'IÖ, Tŭgrul S, Özcan P, et al. Comparison of percutaneous dilatational and forceps guided tracheostomy techniques. Türk Anest Rean Cem Mecmuas. 2001;(29):547-50. Disponible https://www.researchgate.net/publication/51411859_Comparison_of_percutaneous_dilatational_tracheostomy_with_surgic al_tracheostomy

24. Mata J. El papel de la traqueotomía temprana en el paciente críticamente enfermo. Med Int Mex. 2016;32(4):446454.Disponible en: https://www.medigraphic.com/cgi bin/new/resumen.cgi?IDARTICULO=66816

25. Khammas A, Dawood M. Timing of Tracheostomy in Intensive Care Unit Patients. Int Arch Otorhinolaryngol. 2018; 22:437442. Disponible en: https://www.thieme-connect.com/products/ejournals/pdf/10.1055/s-0038-1654710.pdf

26. Tsakiridou E, Mega A, Zakynthinos E, Melissopoulou T, Stamos G, Argyriou K, et al. Pre-intensive care unit intubation and subsequent delayed intensive care unit admission is independently associated with increased occurrence of ventilatorassociated pneumonia. Clin Respir J. 2018;(80). Disponible en:https://pubmed.ncbi.nlm.nih.gov/30015372/

27. Mc Credie V, Alali A, Scales D, Adhikari N, Rubenfeld G, Cuthbertson B, et al. Effect of early versus late tracheostomy or prolonged intubation in critically ill patients with acute brain injury: A systematic review and meta-analysis. Neurocrit Care. 2017; 26:14-25. Disponible en: https://pubmed.ncbi.nlm.nih.gov/27601069/

28. Cai S, Hu J, Liu D, Bai X, Xie J, Chen J et al. The influence of tracheostomy timing on outcomes in trauma patients: A metaanalysis. Injury, Int. J. Care Injured 2017; 48: 866-873. Disponible en: https://www.injuryjournal.com/article/S00201383(17)30094-3/fulltext

29. Khalili H, Paydar S, Safan R, Arasteh P. Experience with Traumatic Brain Injury: Is Early Tracheostomy Associated withBetter Prognosis? World Neurosurg. 2017; 103:88-93. Disponible https://www.sciencedirect.com/science/article/abs/pii/S1878875017302334

30. Sánchez H, Tello A, Pérez R, Aranda R, Aguilar J. Beneficios de la traqueostomia temprana en pacientes con traumatismo craneoencefálico severo. Actual Med. 2019;104(807): 81-85. Disponible en: https://www.actualidadmedica.es/images/807/pdf/or01.pdf 